\title{
Classical Electrodynamics and the Special Relativity Theory
}

\section{Hajra S.}

Calcutta Mathematical Society, India;

E-mail: Hajra <sankarhajra@yahoo.com>;

Electromagnetic fields possess momenta and energies which we could experience with our sense organs. Therefore, those are real physical entities, i.e., objects. All physical objects are subject to gravitation and at the near vicinity of the Earth's surface they are carried with the Earth. Electric and magnetic fields should similarly be subject to gravitation and at the near vicinity of the Earth's surface, they should be similarly carried with the Earth. We shall show in this paper that this simple classical consideration along with classical physics is equivalent to the special relativity theory.

Keywords: Classical electrodynamics, special relativity.

DOI: $10.18698 / 2309-7604-2015-1-187-198$

\section{Introduction}

In this discussion, we shall examine classical electrodynamics and the action of the gravitating field of the Earth on the electric and magnetic fields existing adjacent to the Earth's surface and explain a host of puzzling electrodynamic phenomena easily and rationally from the consideration of classical physics.

\subsection{The field of a steadily moving point charge}

The scalar potential $(\Phi)$ and the induced vector potential $\left(\mathrm{A}^{*}\right)$ of a system of charges (with charge density $\rho$ ) when steadily moves in the OX direction with a velocity u are governed by D' Alembert's equation

$$
\begin{gathered}
\frac{\partial^{2} \Phi}{\partial \mathrm{x}^{2}}+\frac{\partial^{2} \Phi}{\partial \mathrm{y}^{2}}+\frac{\partial^{2} \Phi}{\partial \mathrm{z}^{2}}-\frac{1}{\mathrm{c}^{2}} \frac{\partial^{2} \Phi}{\partial \mathrm{t}^{2}}=-\frac{\rho}{\varepsilon_{0}} \\
\frac{\partial^{2} \mathrm{~A}_{\mathrm{x}}^{*}}{\partial \mathrm{x}^{2}}+\frac{\partial^{2} \mathrm{~A}_{\mathrm{x}}^{*}}{\partial \mathrm{y}^{2}}+\frac{\partial^{2} \mathrm{~A}_{\mathrm{x}} *}{\partial \mathrm{z}^{2}}-\frac{1}{\mathrm{c}^{2}} \frac{\partial^{2} \mathrm{~A}_{\mathrm{x}}^{*}}{\partial \mathrm{t}^{2}}=-\frac{\rho \mathrm{u}}{\varepsilon_{0} \mathrm{c}^{2}}, \mathrm{~A}_{\mathrm{y}}^{*}=0, \mathrm{~A}_{\mathrm{z}}^{*}=0
\end{gathered}
$$

Heaviside solved the equations (1) and (2) directly by using steady state operator and his operational calculus and calculated the fields of a steadily moving point charge $\mathrm{Q}$ at any point, $\mathrm{r}$ distance away from the charge [ $\mathbf{B}^{*}$ and $\mathbf{E}$ through $\mathbf{A}^{*}$ ] in 1888 [1] and then in 1889 [2] as 


$$
E=\frac{Q k^{2} r}{4 \pi \varepsilon_{0} r^{3}}\left(1-\frac{\mathrm{u}^{2}}{\mathrm{c}^{2}} \sin ^{2} \theta\right)^{-3 / 2}, \mathrm{~B}^{*}=u \times E / \mathrm{c}^{2}\left(k=\sqrt{1-u^{2} / c^{2}}, \gamma=1 / k\right)
$$

where $\mathrm{c}$ is the velocity of light in free space, $\varepsilon_{0}$ is the permittivity of free space and $\theta$ is the angle between the direction of motion of the point charge and $\mathbf{r}$.

The fields of a steadily moving point charge were first deduced by Oliver Heaviside in 1888 and not by Einstein as tacitly claimed by the relativists.

From these two relations as given in Eq.(3), electromagnetic momentum(P) of that steadily moving charge could easily be deduced classically from its well known relation with magnetic energy $(\mathrm{T})$ which is as follows:

$$
P=2 \mathrm{Tu} / \mathrm{u}^{2}
$$

Now, from a beautiful calculation of Searle (1897) [3] based on Heaviside's Fields, we have for magnetic energy $(\mathrm{T})$ of a very small spherical charge having radius $\delta \mathrm{R}$ reads,

$$
T=\gamma Q^{2} u^{2} /\left(12 \pi \varepsilon_{0} c^{2} \delta R\right)
$$

Therefore, combining Eqs. (4) \& (5), we have electromagnetic momentum (P) for the steadily moving point charge as shown in $[4,5,6]$ and alternatively in $[7,8,9,10]$,

$$
P=\gamma Q^{2} u /\left(6 \pi \varepsilon_{0} c^{2} 8 R\right)=\gamma m_{0} u, \frac{Q^{2}}{6 \pi \varepsilon_{0} c^{2} \delta R}=m_{0}
$$

The above momentum equation implies that the electromagnetic mass of a point charge varies with velocity.

Similarly, energy of a steadily moving point charge could easily be derived classically as

$$
\left(\text { Energy }=\int \frac{d P}{d t} d x=\gamma m_{0} c^{2}\right)
$$


(a) When the point charge moves rectilinearly with a variable velocity $\mathrm{u}$, the vector $\frac{d \mathrm{P}}{d t}$ is directed along the line of motion and using Eq. (6) its magnitude is given by

(b)

$$
\frac{d P}{d t}=\frac{d P}{d u} \frac{d u}{d t}=\gamma^{3} m_{0} \frac{d u}{d t}
$$

(i) When the charge is moving with a constant velocity of $u$ but of varying direction, the acceleration is then normal to the path and it is convenient to use vector equation. If $\mathrm{u}$ be the velocity and $\frac{d \mathbf{u}}{d t}$ the acceleration and let us take into account that in this case there is a constant ratio between $\mathbf{P}$ and $\mathbf{u}$ and then using Eq. (6) we get

$$
\frac{P}{u}=\gamma m_{0}
$$

From Eqs. (8) \& (9) we have longitudinal electromagnetic mass (LEM) and transverse electromagnetic mass (TEM)of a moving point charge

$$
\begin{gathered}
L E M=\gamma^{3} m_{0} \\
T E M=\gamma m_{0}
\end{gathered}
$$

Thus we see that there are two masses of the steadily moving point charge, i.e., longitudinal and transverse electromagnetic masses that determine the motion of a point charge in electromagnetic fields.

\subsection{Transverse Doppler's Effect}

There is a mainstream propaganda that transverse Doppler's effect could not be explained from classical physics. The propaganda is not correct as we see below:

A dipole radiates when an elastic electromagnetic force acts inside it. When the dipole moves, the fields change inside it. And consequently, frequency and time- period of oscillation of the moving dipole change as per the following classical equations: 
An elastic electric force $\mathbf{F}_{\mathbf{0}}$ acts on the oscillating point charge with rest mass mo inside a stationary radiating dipole having frequency of oscillation $\omega_{0}$ and the amplitude of oscillation $\mathbf{S}$ . Then, as per classical physics

$$
F_{0}=-\mathrm{m}_{0} \omega_{0}^{2} S
$$

But when that dipole-system steadily moves with a velocity $\mathrm{u}$ in the direction perpendicular to its direction of oscillation, the force equation inside the dipole as per classical electrodynamics changes to

$$
F \approx-\gamma m_{0} \omega^{2} S
$$

( $\mathbf{F}$ acting electromagnetic force, ymo transverse electromagnetic mass of the charge moving with the system, $\boldsymbol{\omega}$ frequency and $\mathbf{S}$, amplitude of oscillation in the dipole moving steadily at perpendicular to the direction of oscillation).

Now, when the dipole moves with a velocity $U$ in free space in any direction perpendicular to its direction of oscillations, the electric force and the magnetic force acting on the charge will be respectively $\gamma \mathbf{F}_{0}$ and $-\left(\mathrm{u}^{2} / \mathrm{c}^{2}\right) \gamma \mathbf{F}_{0}$ (from Eq. (3) of Heaviside). Therefore, total electromagnetic force acting between these two moving charges from Heaviside's field and Lorentz Force Law is

$$
F=\gamma \mathrm{F}_{0}-\left(\mathrm{u}^{2} / \mathrm{c}^{2}\right) \gamma \mathrm{F}_{0}=F_{0} \sqrt{1-\mathrm{u}^{2} / \mathrm{c}^{2}}=F_{0} k
$$

Comparing Eqs. (12), (13) and (14) for the dipole moving with an uniform velocity $\mathrm{u}$ in any direction perpendicular to its direction of motion, we have,

$$
\omega=\omega_{0} \sqrt{1-u^{2} / c^{2}}
$$

This explains transverse Doppler's effect classically.

\subsection{The So-called Time Dilation}

Now if the frequency changes, time period too changes.

For a radiating dipole stationary in free space the time period of oscillation is given by 


$$
t_{0}=2 \pi / \omega_{0}
$$

For the dipole steadily moving perpendicular to its direction of oscillation, the time-period of oscillation is given by

$$
t=2 \pi / \omega
$$

Comparing Eq. (16) with (17) using eq. (15)

$$
t=\gamma t_{0}
$$

or the period of oscillation of the above moving dipole increases with its velocity in free space.

\subsection{Increment of Life Spans of Moving Radioactive Particles}

There is also a mainstream propaganda that increment of life-spans of moving radioactive particles could not be explained from classical physics. The propaganda too is not correct as we see below:

A radioactive particle decays when electric and magnetic forces inside the particle act to disintegrate the particle. When the radioactive particle moves, the electric and magnetic forces acting inside the particle decrease. And consequently, the disintegration process in the moving radioactive particle decreases and life span of the particle increases as per the following classical equations:

In such a situation, the following equation represents the relation between initial untransformed radioactive particle $\left(\mathrm{N}_{0}\right)$ and the untransformed radioactive particles $(\mathrm{N})$ after the time to in a stationary system of decaying radioactive particles.

$$
\mathrm{N}=\mathrm{N}_{0} \mathrm{e}^{-\lambda \mathrm{F}_{0} \mathrm{t}_{0}}
$$

where the disintegrating force inside any particle is $\mathbf{F}_{0}$.

Suppose that the radioactive particles of the above system are polarized in a certain direction and are made to move in a direction perpendicular to the direction of the acting force inside the particle with a velocity $\mathrm{u}$. Then the following equation represents the relation between 
initial untransformed radioactive particle $\left(\mathrm{N}_{0}\right)$ and the untransformed radioactive particles $(\mathrm{N})$ after the time $t$

$$
\mathrm{N}=\mathrm{N}_{0} \mathrm{e}^{-\lambda F \mathrm{t}}
$$

where the disintegrating force inside any particle is $\mathbf{F}$ due to motion.

Comparing Eq. (19) and eq. (20) using Eq. (14), we have

$$
t=\gamma t_{0}
$$

i.e., life spans of radioactive particles increase with velocity.

This analysis at once destroys 'here is one time', 'there is another time'-concept as well as the twin paradox of relativity as when the observer moves and the particle is at rest on the surface of the earth there will be no life-span increment.

\subsection{Fizeau Experiment}

The result of Fizeau experiment has already been explained by Lorentz with the introduction of Polarization vector in the Maxwell equation for light propagating through a steadily moving dielectric.

The equation of Polarization $\boldsymbol{p}$ for a dielectric moving with velocity $\mathbf{v}$ in the free space could be written in terms of the electric field vector $\mathbf{E}$ Eand magnetic field vector $\mathbf{B}$ as follows $[11,12]$ :

$$
\begin{aligned}
& p=\varepsilon_{0}\left(n^{2}-1\right) E^{\prime} \\
& E^{\prime}=E+V \times B
\end{aligned}
$$

where $\varepsilon_{0}$ is the permittivity of the free space and $n=\sqrt{K}$ where $K$ being dielectric constant of the medium.

Therefore, using Eqs. (22) \& (23), we have,

$$
p_{x}=\varepsilon_{0}\left(n^{2}-1\right)\left(E_{x}-v B_{y}\right)
$$


Now let the axis of $z$ be taken parallel to the direction of motion of the dielectric, which is supposed to be direction of propagation light. Consider a plane polarized wave. Let the axis of $x$ parallel to the Electric field so that magnetic field parallel to the axis of $y$.

Now the fundamental equations in this system will take the following forms:

$$
\begin{gathered}
\nabla \times H=\frac{\partial D}{\partial t}=>-\frac{\partial H_{y}}{\partial z}=\frac{\partial D_{x}}{\partial t}+V \frac{\partial p_{x}}{\partial z} \\
\nabla \times E=-\frac{\partial B}{\partial t} \Rightarrow>-\frac{\partial E_{x}}{\partial z}=\frac{\partial B_{y}}{\partial t}
\end{gathered}
$$

Eliminating $D_{x}, p_{x}, H_{y}$ and neglecting $v^{2} / c^{2}$, we have

$$
\frac{\partial^{2} E_{x}}{\partial z^{2}}=\frac{n^{2}}{c^{2}} \frac{\partial^{2} E_{x}}{\partial t^{2}}+\frac{2 V}{c^{2}}\left(n^{2}-1\right) \frac{\partial^{2} E_{x}}{\partial t \partial z}
$$

Substituting

$$
E_{x}=e^{n(t-z / V) \sqrt{-1}}
$$

where $V$ denotes the velocity of light in the moving dielectric with respect to free space. Therefore

$$
c^{2}=n^{2} V^{2}-2 V\left(n^{2}-1\right) V
$$

Neglecting $v^{2} / c^{2}$, we get

$$
V=\frac{c}{n}+\left(1-\frac{1}{n^{2}}\right) V
$$

The factor associated with $v$ in the right hand part of the equation is Fresnel drag coefficient verified by Fizeau Experiment [5]. For alternative deduction vide [7, 8, 9, and 10] 


\subsection{Puzzling Electrodynamic Phenomena}

Maxwell's equations of electromagnetic fields are applicable only in free space and inside systems stationary in free space. One would then expect some corrections/ modifications of Maxwell's equations when the electromagnetic phenomena are studied on the surface of the earth which is moving with a high velocity in the free space. But those corrections are not needed!

All electrodynamic phenomena like reflection, refraction, diffraction, interference etc., as observed on the surface of the earth, either with star light or with earth light are independent of the movement of this planet. That is: the earth's surface is exactly equivalent to free space for our description of electromagnetic phenomena on it.

Electric and magnetic fields possess momenta and energies which we could experience with our sense organs. Therefore, electric and magnetic fields are real physical entities (objects) [13]. All physical objects are subject to gravitation. They are carried with the Earth at the near vicinity of the Earth's surface. They spin, translate and rotate, too, with the Earth at its surroundings. The electric and magnetic fields should similarly be subject to gravitation and should similarly be carried with the Earth at its near vicinity. They should similarly spin, translate and rotate, too, at the surroundings of this planet. Electric and magnetic fields originating either from the Earth, or the sun, or from the stars, existing at the near vicinity of the earth's surface, should be carried with the Earth, and should spin, translate, and rotate with the earth, exactly in same way as other physical objects do. This will at once explain all puzzling electrodynamic phenomena easily and rationally $[5,7,8,9$, and 10$]$.

\section{(i)The Trouton-Noble Experiment (1904)}

In a laboratory, when a charged condenser moves, the electric field around it changes and thereby a magnetic field is created around the condenser. If the electric field originating from the condenser would move along with the condenser, there would be no change of electric field around the condenser and thereby, there would be no magnetic field around it.

Now, a condenser at rest on the earth's surface moves with the Earth. But the electric field around the condenser, too, moves with it. And therefore, there should be no magnetic field around that condenser when it is stationary on the surface of the Earth which is moving with a high velocity in free space. Therefore, the Trouton-Noble Experiment (1904) fails to detect any magnetic field around the condenser. This implies that the earth carries the condenser along with its electric field.

\section{(ii)The Michelson-Morley type Experiments in air and water}


The Earth translates along with air, water and electromagnetic fields at the vicinity of its surface. Therefore, the motion of the Earth should have no effect on the velocity of light in air or water when measured on the surface of the Earth. This will at once explain the null results of all the Michelson-Morley type Experiments in air and the Mascart-Jamin type Experiments in water at rest on the Earth's surface; and may give us some insight to understand why all electromagnetic phenomena as observed on the surface of the earth are independent of the motion of this planet.

\section{(iii)The Kennedy - Thorndike experiment and the Michelson-Gale-Pearson}

\section{Experiment}

When the Earth translates, spins and rotates in its orbit, air, water and electromagnetic fields at the vicinity of its surface translate, spin and rotate with it . Therefore, all these motions should have no effect on the velocity of light in air or water stationary on the surface of the Earth excepting a minute effect for the Coriolis forces on the light beams. In the Kennedy - Thorndike experiment, it is observed that the velocity of light on the surface of the earth is independent of translation and spinning of the earth around its axis and the Michelson- Gale Experiment proves that there is Coriolis effect on the circuital light beams propagating near the Earth's surface.

\section{(iv)The Tomaschek (1924) and Miller's Experiment (1925)}

where the Michelson-Morley experiment has been performed with starlight and sunlight, similar null results have been confirmed.

This can only happen if the electric and magnetic fields originating either from the earth, stars or from the Sun and existing at the near vicinity of the earth's surface, spin, translate and rotate with the earth.

\section{(v)Sagnac Experiment}

As per classical electrodynamics, light signals, divided in two parts and sent in opposite directions around a fixed circuit on a spinning disk, should not return to the point of division at the same instant. Because, the speed of light on a spinning disk is $\mathrm{c}-\mathrm{v}$ hen the light beam travels towards the direction of spinning of the disk, and $\mathrm{c}+\mathrm{v}$ when the light beam travels in the opposite direction, $\mathrm{v}$ being the spinning velocity of the point on the disk where the speed of light is being measured. The actual experiment confirms this. This effect of light on a spinning disk was observed by G. Sagnac in an interferometer fixed on the disk in 1913 and is known as the Sagnac effect.

$$
\Delta t=2 \pi R\left(\frac{1}{c-V}-\frac{1}{c+V}\right)=\frac{4 A \Omega}{c^{2}}
$$




$$
\Delta f=\frac{4 A \Omega}{c \lambda}
$$

$(\Delta \mathrm{t}=$ time difference, $\Delta \mathrm{f}=$ fringe shift, $\mathrm{R}=$ radius of the disk, $\Omega$ =angular velocity of the disk, $\mathrm{A}$ $=$ area covered by the ray circuit)

It is now revealed that F. Harress performed a similar experiment in 1911 . "Harress had both the photographic equipment and the light source fixed in the laboratory, whereas Sagnac had both on the spinning disk". Dufour \& Prunier made similar tests when the photographic plate was on the disk but the source of light was in the laboratory and vice-versa. The fringe shift followed the Sagnac formula in all the cases. The effect is the same for ring laser and light propagated through optical fibers. Many workers have reported similar effects for electrons and neutrons. Many people believe that Sagnac effect is not consistent with SRT. Relativists present many motivated explanations on the phenomena to save their absurd propositions.

Sagnac Experiment proves that the speed of light is relative.

Sagnac effect on the Earth is not affected by the spinning and translation of the Earth.

(vi)The Observations of Bradley (1728), Airy (1871) and Zapffe (1992) on aberration of light

\section{Aberration of astral and terrestrial light}

a) Suppose a ray from an overhead fixed star is coming to the earth which is moving with respect to the fixed star with a velocity $u$.

If a light beam is not influenced by the gravitating field of the Earth, from the consideration of the relative velocity of classical physics, the man on the earth should see the star not on overhead through a telescope. Instead he should see the star deflected at an angle $\theta$ towards the direction of motion of the earth from overhead such that $\tan \theta=\frac{u}{c}$ where $\mathrm{u}$ is the velocity of the earth with respect to the fixed star and $\mathrm{c}$ is the velocity of the ray in space fixed with the fixed star. Now if the telescope be filled with water, the man should see the star at an angle $\theta_{1}$ (such that $\tan \theta_{1}=\frac{n u}{c}$ ) deflected towards the direction of motion of the earth from overhead, $\mathrm{n}$ being the refractive index of water.

In this case, the ray velocity and the phase velocity of the wave coming from the star will be different. The direction of the ray is here is the apparent direction and a ray coming from a mountain top should have the same aberration as given in the above analysis. For, the ray as per 
Maxwell should propagate with a velocity c with respect to free space which could be conceived as fixed with the fixed stars. Observations do not corroborate this explanation.

b) Now if the stars and the planets carry electric and magnetic fields along with them at their surroundings, a ray from an overhead fixed star will be carried with the star at its vicinity, and then it will enter the galactic space and will be carried with the galaxy and then it will reach the surrounding of the sun and it will be carried with the sun. Then it will proceed and strike the electric and magnetic field coverage of the Earth at the near vicinity of its surface at an angle

$\theta$ deflected towards the direction of the motion of the earth such that $\tan \theta=\frac{u}{c}$ and the ray will be carried with the earth, ' $u$ ' being the velocity of the earth with respect to the sun and 'c' is the velocity of light in the solar space. The ray and its direction here are real.

On the surface of the Earth, in this case, there is no relative motion between the ray and the Earth . Therefore, a man on earth will see the star with an angle $\theta$ tilted towards the direction of motion of the Earth (as was observed by Bradley). If he fills the telescope with water, the ray velocity inside water must be $c / n$. But as there is no relative velocity between the ray inside water and the Earth, the position of the star will not alter ( i.e, there will be no further aberration as observed by Airy). Here the ray velocity and the phase velocity are the same and the ray and its direction in both the instances are real.

More interestingly, in such a situation, a ray coming from a mountain top should have no aberration as per Zapffe's (1992) report [14]. Observations corroborate this explanation.

\section{Conclusion:}

Our study shows that all special relativistic phenomena could easily and rationally be explained from the consideration of classical physics and thereby exposes the uselessness of the special relativity theory in the domain of electrodynamics.

Acknowledgement:

Your author gratefully acknowledges the grant provided to him by Dr. M. C. Duffy, Founder Secretary of PIRT-London to present this paper personally in the PIRT-Moscow 2015 conference.

Your author was encouraged by the works of Debabrata Ghosh [15], Reserve Bank of India, Calcutta and Prof. K.C. Kar [16], Editor, Indian Journal of Theoretical Physics to write this paper. 


\section{References}

1. Heaviside O. (1888). The Electromagnetic Effect of Moving Point Charge. The Electrician, 22 147-148.

2. Heaviside O. (1889). On the Electromagnetic Effects due to the Motion of Electricity through a Dielectric. Phil. Mag., 27, 324-339.

3. Searle G.F.C. (1897). On the Steady Motion of an Electrified Ellipsoid. The Phil. Mag,. 44, 329341.

4. Hazra K. (2008). On the Resolution of Twin Paradox. Current Science, 95 (6), 706-707.

5. Hajra S. (2011). Cross Radial Force. Proceedings NPA, Vol. 8, 235-240.

6. Hajra S. (2014). Classical Interpretations of relativistic precessions. Chin, Phy., B 23 (4), 40402-.

7. Hajra S., Ghosh D. (2000). A Critical Analysis of Special Relativity. Proceedings PIRT-London, 137-158.

8. Hajra S. (2005). Collapse of SRT 1: Derivation of Electrodynamic Equations from Maxwell Field Equations. GED, 15 (4), 63-70.

9. Hajra S. (2003). Nature of Electric and Magnetic Fields at the vicinity of the Earth's surface. Proceedings, PIRT-Moscow, 224-238.

10. Hajra S. (2012). Classical Interpretations of Relativistic Phenomena. Jour. Mod. Phy., 3 (2), 187-199.

11. Whittaker E.T. (1951). A History of the Theories of Aether and Electricity. London: Thomas, Nelson and Sons Limited.

12. Tamm I.E. (1961). Fundamentals of the Theory of Electricity. Moscow: MIR [World]. 583.

13. Kompaneyets A.S. (1961). Theoretical physics. Moscow: Foreign Language Publishing House, 105.

14. Zapffe C.A. (1992). Bradley Aberration and Einstein Space Time. Ind. J. of Theo. Phys., 40, 145-148.

15. Ghosh D. (1994). In the Background of the Michelson- Morley Experiment. Ind. Jour. Theo. Phy., 42 (1), 73-79.

16. Kar K.C. (1970). A New Approach to the Theory of Relativity. 